\title{
Global burden of neuroendocrine tumors and changing incidence in Kentucky
}

\author{
Aman Chauhan ${ }^{1,2}$, Qian Yu ${ }^{1}$, Neha Ray ${ }^{3}$, Zainab Farooqui ${ }^{4}$, Bin Huang ${ }^{1,5}$, Eric B. \\ Durbin $^{1,2}$, Thomas Tucker $^{1,6}$, Mark Evers ${ }^{1,7}$, Susanne Arnold ${ }^{1,2}$ and Lowell B. \\ Anthony ${ }^{1,2}$ \\ ${ }^{1}$ Markey Cancer Center, University of Kentucky, Lexington, KY, USA \\ ${ }^{2}$ Department of Internal Medicine, University of Kentucky, Lexington, KY, USA \\ ${ }^{3}$ University of Cincinnati School of Medicine, Cincinnati, Ohio, USA \\ ${ }^{4}$ Kentucky College of Osteopathic Medicine, Pikeville, KY, USA \\ ${ }^{5}$ Department of Biostatistics, University of Kentucky, Lexington, KY, USA \\ ${ }^{6}$ Kentucky Cancer Registry, Lexington, KY, USA \\ ${ }^{7}$ Department of Surgery University of Kentucky, Lexington, KY, USA \\ Correspondence to: Aman Chauhan, email: Amanchauhan@uky.edu \\ Keywords: neuroendocrine tumor incidence; kentucky cancer registry; SEER \\ Received: January 10,2018 Accepted: March 15, $2018 \quad$ Published: April 10, 2018 \\ Copyright: Chauhan et al. This is an open-access article distributed under the terms of the Creative Commons Attribution License \\ 3.0 (CC BY 3.0), which permits unrestricted use, distribution, and reproduction in any medium, provided the original author and \\ source are credited.
}

\section{ABSTRACT}

Background: Neuroendocrine tumors (NETs) have a low incidence but relatively high prevalence. Over the last three decades, the incidence of NETs has risen 6 -fold in the United States. We conducted an observational study to compare the incidence of NETs reported to the Kentucky Cancer Registry (KCR) versus that reported to Surveillance, Epidemiology, and End Results Program (SEER). We also provide a systematic review of the state of neuroendocrine tumors worldwide, and compare the available global and local published data.

Methods: KCR and SEER databases were queried for NET cases between 1995 and 2015. A detailed literature review of epidemiological data for various nations worldwide summarize epidemiological data from various countries.

Results: KCR recorded 6179 individuals with newly diagnosed NETs between 1995 and 2015. Between 1995-2012, the incidence of NETs in KCR increased from 3.1 to 7.1 per 100,000 cases, while it increased from 3.96 to 6.61 in the SEER database. The incidence rates in both KCR and SEER databases were linear. $\mathbf{9 0 . 5 7 \%}$ were Caucasians with $54.74 \%$ females. $27.67 \%$ of the Kentucky population was from the Appalachian region. Patients aged 50-64 years had the highest prevalence $(38 \%)$. Lung NET ( $30.60 \%)$ formed the bulk of cases, followed by small intestine $(16.82 \%)$, rectum/anus $(11.35 \%)$ and colon $(9.71 \%)$.

Conclusions: NETs incidence between 1995 and 2015 show a linear increase in both KCR and SEER databases. Because of this increased incidence it is imperative for community oncologists to familiarize themselves with this entity, which until recently was under-studied and with few viable treatment options. 


\section{INTRODUCTION}

Neuroendocrine tumors are frequently grouped with other rare malignancies, which limits drug development and clinical trial expansion for this important oncologic entity. Fortunately, the last 15 years have seen rapid changes in awareness and perception of general oncologists toward neuroendocrine tumors. As a result, there is not only an expansion of evidence-based standard of care data for the treatment of these patients, but future therapeutic drug developmental avenues also look promising. Robust epidemiological surveillance of this population helps us recognize the quantum of disease burden and unique patterns of disease presentation, which are key in the use of effective screening, targeted diagnostics, and treatments.

We performed a retrospective analysis of the Kentucky Cancer registry (KCR) and the Surveillance, Epidemiology, and End Results Program (SEER) databases between 1995 and 2015. Studies outside of the U.S. were also analyzed in terms of incidence, anatomical sites, grade, and survival. The aim of the study is to analyze epidemiologic and demographic data of newly diagnosed neuroendocrine tumors in Kentucky and compare this information with currently available global data.

\section{RESULTS}

We identified 6179 individuals with newly diagnosed NET between 2005 and 2015. We observed a steady rise in annual incidence of NET from 119 new cases in 1995 to 528 cases in 2015 (Table 1). After accounting for Kentucky's population increase (from 4.183 million to 4.425 million during those years), we determined that the corresponding incidence rate increased from 3.1 to 10.3 per 100,000 population (Table 1). Of our study patients, $90.57 \%$ were Caucasian with $54.74 \%$ females. Patients aged 50-64 years had the highest prevalence (38\%). Geographically, $27.67 \%$ of our Kentucky population was from the Appalachian region. Lung NET (30.60\%) formed the bulk of cases, followed by small intestine $(16.82 \%)$, rectum/anus (11.35\%) and colon $(9.71 \%)$ (Table $2 \mathrm{~A}$ ). $46.92 \%$ of females presented with localized disease as compared to $36.07 \%$ males. $26.45 \%$ of African Americans NET patients were aged between $20-49$ years as compared to $18.22 \%$ of Caucasian patients. Lung NET was highest among smokers, whereas small bowel NET was highest among nonsmokers.

The majority of cases (52\%) were low grade, with $22 \%$ high grade, $3 \%$ intermediate grade, and the rest unknown (Table 2B). Grading of tumors is based on the globally-accepted WHO criteria, which considers Ki 67 $<2 \%$ low grade, $2-20 \%$ intermediate grade, $>20 \%$ high grade [1]. This grading system might be modified in the future based on recent data from Tang et al. [2] It should be noted that roughly $25 \%$ of newly diagnosed NET patients are histologically placed at either G2 or G3. These patients have a distinct clinicopathological course as compared to low grade (G1) NETs.

Unlike most other malignancies, NETs are usually diagnosed at an early stage. In our study population, $42 \%$ had local disease at the time of diagnosis, $22 \%$ had locoregional disease, $22 \%$ presented with distant metastatic disease, and the other $14 \%$ had an unknown status. Except for those with local disease, where $25 \%$ were females as compared to $16 \%$ males, the loco-regional and metastatic subgroups did not show any gender discrepancy. Staging has a profound impact on the choice of treatment, with early stage being curative with surgical resection alone, especially for low grade histology. As expected, 52\% of our study population underwent surgery alone as the treatment of choice.

As far as age stratification is concerned, peak incidence was found in 50-64 year age group (38\%), followed by $65-74$ (25\%), 20-49 (19\%) and 75+ (18\%). These data are in agreement with most found in the published literature.

Lastly, $27 \%$ of our study population reside in the Appalachian region. Multivariate analysis of the Appalachian population did not show significant aberration in patterns compared with the non-Appalachian population with regard to any of the previously discussed study variables.

\section{DISCUSSION}

Derived from the neural crest cells during development, NETs can arise from various primary sites in the body. Neuroendocrine tumors are commonly found in the gastrointestinal tract but it is not unusual to find neuroendocrine tumors in lungs, pancreas, thyroid, gonads, and other locations [3-5]. Besides site of origin, NETs can also be subdivided between secretory or nonsecretory subgroups based on production of hormones or into high grade, intermediate grade or low-grade tumors based on the pathology. There is substantial heterogeneity among all these subgroups in terms of natural history of disease progression, response to therapeutic agents, and overall survival. Despite their low incidence rate, all NETs harbor the potential for malignancy, rendering the term "carcinoid tumor," adopted by previous researchers, inaccurate. In addition to their misnomer, the diagnosis criteria and classifications of NETs have been under debate since their discovery at the beginning of the last century. JACC, WHO, and ENETS criteria standards differ among each other, and the criteria for NETs from various sites may also vary within the same guideline. Along with the lack of population-based NET registries across the world in general, these variances have prevented us from elucidating the worldwide epidemiology of NETs over time. Rather, a multitude of studies emerged to assess 
Table 1: KCR and SEER NETs cases, 2005-2015

\begin{tabular}{|c|c|c|c|c|}
\hline Year & $\begin{array}{c}\text { KCR } \\
\text { (age adjusted } \\
\text { incidence) }\end{array}$ & $\begin{array}{c}\text { SEER } \\
\text { (age adjusted } \\
\text { incidence) }\end{array}$ & $\begin{array}{c}\text { KCR } \\
\text { (Cases) }\end{array}$ & $\begin{array}{c}\text { SEER } \\
\text { (Cases) }\end{array}$ \\
\hline 1995 & 3.1 & 3.96 & 119 & 1299 \\
\hline 1996 & 3.6 & 4.12 & 138 & 1372 \\
\hline 1997 & 3.6 & 4.29 & 141 & 1456 \\
\hline 1998 & 4.7 & 4.73 & 188 & 1631 \\
\hline 1999 & 5 & 4.76 & 204 & 1671 \\
\hline 2000 & 5.3 & 4.9 & 218 & 3613 \\
\hline 2001 & 5.2 & 4.67 & 214 & 3510 \\
\hline 2002 & 5.5 & 5.14 & 232 & 3947 \\
\hline 2003 & 5.2 & 5.29 & 226 & 4123 \\
\hline 2004 & 5.9 & 5.44 & 257 & 4335 \\
\hline 2005 & 5.8 & 5.56 & 257 & 4378 \\
\hline 2006 & 6.3 & 5.94 & 281 & 4880 \\
\hline 2007 & 7.4 & 6.16 & 342 & 5196 \\
\hline 2008 & 7.4 & 6.27 & 351 & 5401 \\
\hline 2009 & 7.2 & 6.38 & 346 & 5628 \\
\hline 2010 & 8.2 & 6.57 & 405 & 5913 \\
\hline 2011 & 7.1 & 6.61 & 351 & 6042 \\
\hline 2012 & 8.4 & & 414 & \\
\hline 2013 & 8.6 & & 447 & \\
\hline 2014 & 10.4 & & 520 & \\
\hline 2015 & 10.3 & & 528 & \\
\hline
\end{tabular}

Table 2A: Site-specific prevalence of neuroendocrine tumors in Kentucky

\begin{tabular}{lc}
\hline Site & Prevalence (\%) \\
\hline Lung & 30.6 \\
Small Intestine & 16.82 \\
Rectum/Anus & 11.35 \\
Colon & 9.71 \\
Pancreas & 5.5 \\
Others & 12.91 \\
Unknown & 7.61 \\
\hline
\end{tabular}


Table 2B: Grade specific distribution of NETs in Kentucky

\begin{tabular}{lc}
\hline WHO 2010 Grading & $\%$ \\
\hline G1 & 52 \\
G2 & 3 \\
G3 & 22 \\
Unknown & 23 \\
\hline
\end{tabular}

the prognostic value of each classification scheme. In this systematic review, we examined NET cohort studies by country, focusing on the profile of NET composition and incidences, to gain a worldwide perspective of NET epidemiology.

\section{Primary sites}

Consistent with the SEER database, the most common NET sites in KCR database were lung, small intestine, and rectum-anus (Table 2A) [6]. A nation-wide study from Netherland also reported pulmonary NET being the most prevalent. In contrast, two studies from Denmark and Sweden reported small intestine NETs to be most prevalent $[7,8]$. If only gastroenteropancreatic neuroendocrine tumors (GEP-NETs) were considered, studies in Argentina, France, and Norway reported the small intestine as being the most common anatomical location [9-11]. Table 3 summaries the three most prevalent NET sites globally. Appendix NET was prevalent among Western countries in general, which could be explained by accidental findings during appendectomy. The survival rates of appendix NET were also the most optimal, which might also result from early detections (Table 4). In contrast, rectal NET was particularly common in Asian populations [12-15]. Whether these distinctions resulted from genetic variations needs further research.

\section{Grading and survival}

From a global perspective, few countries reported grading data according to $\mathrm{Ki}-67$ and/or mitotic counts due to the continuously evolving grading systems. The percentages of each grade varied among studies. Although a nation-wide, multicenter study in Korea reported a high G1 percentage of $92.3 \%$, a regional study in China indicated that $73.9 \%$ of all graded cases were G3. Nevertheless, a consistent impression among all studies was that lower grade NETs demonstrated a better outcome in terms of survival. Tables 4 and 5 summarize global NET 5 -year overall survival by site and by grade. Grade 1, Grade 2, and Grade 3 in our Kentucky population were $67.5 \%, 28.6 \%$, and $3.9 \%$, respectively (Table $2 \mathrm{~B}$ ). Table 6 summarizes global prevalence of NETs by grade. Our data underscores the fact that roughly $25 \%$ of newly diagnosed
NET patients in Kentucky present with either G2 or G3 histology. This is a significant number considering these intermediate grade and high grade NETs are often aggressive and have very limited treatment options.

\section{Incidence}

Unlike most malignancies, NET incidence has shown a constant incline. The cause of this increase is not entirely clear but experts have attributed the pattern to an increased awareness and improved diagnostics. The hike in incidence of this otherwise rare tumor has alerted public health stakeholders, which has resulted in establishment of specialized NET registries worldwide [16, 17]. A seminal paper from Yao et al. showed a 5-fold increase in incidence of NET from 1973 to 2004 [3]. Incidence of NET per Yao's report is estimated to be 5.25 cases per 100,000 population. Similar observations were made by Tsikitis et al. in the GI NET subgroup [18]. Per their report, overall incidence of GI NETs increased in all sites except for appendix tumors. In Germany, the age-standardized incidence increased from 2.7 to 3.4 fold from 1976 to 2006 [19]. In Italy, the incidence increased from 0.7 to 5.3 between 1976 and 2010 [20]. Based on recently published papers, increasing trends were also observed in China Japan, Korea, Norway, Netherlands, and Taiwan [11-15, 21]. Outside of the United States, incidence rates ranged from 1.41 to 5.86 based on studies published after 2000 [13, 15, 19, 20, 22-25]. Based on our analysis, the incidence of NETs reported in Kentucky according to the KCR database increased from 3.1 (1995) to 10.3 (2015) per 100,000 cases. In contrast, according to SEER data, the incidence increased from 3.9 to 6.61 between 1995 and 2012 (Figure 1).

\section{Limitations}

Since the beginning of the last century, the nomenclature, diagnostic criteria and classification of NETs have been evolving. "Carcinoid tumor", a previous term adopted by several early studies, was inaccurate because all NETs harbor the potential for malignancy [7, 26-30]. Older versions of the International Classification of Disease for Oncology excluded benign NETs. The WHO has changed grading criteria over time. All of these inconsistencies prevent achieving a clear comparison of 
Table 3: Top 3 primary sites of NETs, reported by country

\begin{tabular}{|c|c|c|c|c|c|c|}
\hline Country & Authors & Years & Sample size & $\begin{array}{l}1^{\text {st }} \text { Most Common } \\
\text { Primary Site }\end{array}$ & $\begin{array}{c}2^{\text {nd }} \text { Most } \\
\text { Common } \\
\text { Primary Site }\end{array}$ & $\begin{array}{c}3^{\text {rd }} \text { Most Common } \\
\text { Primary Site }\end{array}$ \\
\hline Argentina & [9] & NA & $\begin{array}{c}532 \\
\text { (GEP-NET only) }\end{array}$ & $\begin{array}{l}\text { Small Intestine } \\
(26.9 \%)^{1}\end{array}$ & Pancreas $(25.2 \%)$ & $\begin{array}{l}\text { Colon-Rectum-Anus } \\
\qquad(12.4 \%)\end{array}$ \\
\hline $\begin{array}{l}\text { Austria } \\
\text { (prospective } \\
\text { study) }\end{array}$ & {$[22]$} & $2004-2005$ & 265 & Stomach $(23 \%)$ & Appendix (21\%) & Small Intestine $(15 \%)$ \\
\hline Brazil & {$[31]$} & 1997-2009 & $\begin{array}{c}773 \\
\text { (GEP-NET only) }\end{array}$ & Stomach $(24.5 \%)$ & $\begin{array}{l}\text { Small Intestine } \\
(20.8 \%)\end{array}$ & Rectum $(20.5 \%)$ \\
\hline Canada & [23] & 1994-2009 & 5619 & Pancreas $(25 \%)$ & $\begin{array}{c}\text { Colon } \\
(22.8 \%)\end{array}$ & $\begin{array}{l}\text { Small Intestine } \\
\qquad(21.6 \%)\end{array}$ \\
\hline Canada & {$[25]$} & $1990-2005$ & 530 & $\begin{array}{l}\text { Small Intestine } \\
(55.3 \%)\end{array}$ & $\begin{array}{l}\text { Colon } \\
(18.3 \%)\end{array}$ & Appendix (17.9\%) \\
\hline China & [13] & 2009-2013 & $\begin{array}{c}248 \\
\text { (GEP-NET only) }\end{array}$ & Rectum (30.6\%) & Pancreas $(23.4 \%)$ & Gastric $(13.3 \%)$ \\
\hline China & {$[32]$} & $1991-2013$ & $\begin{array}{c}130 \\
\text { (NET with liver } \\
\text { metastasis only) }\end{array}$ & Pancreas $(65.4 \%)$ & Stomach $(10.8 \%)$ & Small Intestine $(5.4 \%)$ \\
\hline China & {$[33]$} & 2011-2016 & $\begin{array}{c}440 \\
\text { (GEP-NET only) }\end{array}$ & Stomach $(24.3 \%)$ & Rectum (24.1\%) & Pancreas $(20.5 \%)$ \\
\hline $\begin{array}{l}\text { China } \\
\text { (Hongkong) }\end{array}$ & [34] & 1994-2013 & $\begin{array}{c}126 \\
\text { (GEP-NET only) }\end{array}$ & Pancreas $(34.9 \%)$ & Rectum $(33.3 \%)$ & Stomach $(8.7 \%)$ \\
\hline Denmark & {$[7]$} & 1978-1989 & 1029 & $\begin{array}{l}\text { Small Intestine } \\
(29.4 \%)\end{array}$ & $\begin{array}{c}\text { Appendix } \\
(17.9 \%)\end{array}$ & Pulmonary (16.8\%) \\
\hline France & {$[10]$} & 2001-2002 & $\begin{array}{c}668 \\
\text { (GEP-NET only) }\end{array}$ & $\begin{array}{l}\text { Small Bowel and } \\
\text { Colon }(43 \%)\end{array}$ & Pancreas $(32 \%)$ & Gastric $(5 \%)$ \\
\hline Germany & {$[35]$} & 2004-2007 & 1263 & Pancreas (31\%) & $\begin{array}{l}\text { Small Intestines } \\
\qquad(22 \%)\end{array}$ & $\begin{array}{l}\text { Colon and Rectum } \\
\qquad(11.4 \%)\end{array}$ \\
\hline Italy & {$[36]$} & 2004-2007 & 1203 & Pancreas $(31 \%)$ & $\begin{array}{l}\text { Lung } \\
(29 \%)\end{array}$ & $\begin{array}{l}\text { Ileum, Cecum, Colon, } \\
\text { Rectum }(13 \%)\end{array}$ \\
\hline Korea & {$[14]$} & 2000-2009 & $\begin{array}{l}4951 \text { (GEP-NET } \\
\text { only) }\end{array}$ & Rectum (48\%) & Stomach $(14.6 \%)$, & Colon $(7.9 \%)$ \\
\hline Lebanon & {$[37]$} & 2001-2012 & $\begin{array}{c}89 \\
(\text { GEP-NET) }\end{array}$ & Pancreas $(24.7 \%)$ & Stomach $(20.8 \%)$ & Duodenum (18.2\%) \\
\hline Mexico & {$[38]$} & NA & $\begin{array}{l}495 \text { (GEP-NET } \\
\text { only) }\end{array}$ & Pancreas (33.27\%) & $\begin{array}{l}\text { Stomach } \\
(28.02 \%)\end{array}$ & NA \\
\hline Norway & {$[24]$} & 1993-2004 & 2030 & $\begin{array}{l}\text { Small Intestine } \\
(25.5 \%)\end{array}$ & $\begin{array}{c}\text { Lung and } \\
\text { Bronchus }(21 \%)\end{array}$ & Colon $(8 \%)$ \\
\hline Norway & {$[11]$} & $2003-2013$ & $\begin{array}{c}204 \text { (GEP-NET } \\
\text { only) }\end{array}$ & $\begin{array}{l}\text { Small Intestines } \\
(29.4 \%)\end{array}$ & $\begin{array}{l}\text { Appendix } \\
(23.5 \%)\end{array}$ & Pancreas (16.2\%) \\
\hline Netherlands & {$[21]$} & $2001-2010$ & 24759 & Pulmonary $(72.7 \%)$ & Appendix (3.9\%) & Small Intestine (3.7\%) \\
\hline Spain & [39] & 2001-2008 & 907 & Pancreas $(34 \%)$ & $\begin{array}{l}\text { Jejunum-Ileum } \\
(15.6 \%)\end{array}$ & Appendix (9.4\%) \\
\hline Sweden & [8] & $1987-2012$ & 7334 & $\begin{array}{l}\text { Small Intestine } \\
(32 \%)\end{array}$ & Appendix (23\%) & $\begin{array}{l}\text { Lung } \\
(15 \%)\end{array}$ \\
\hline Taiwan & {$[15]$} & 1996-2008 & 2187 & Rectum (25.4\%) & $\begin{array}{l}\text { Lung/Bronchus } \\
\qquad(20 \%)\end{array}$ & Stomach $(7.4 \%)$ \\
\hline $\begin{array}{l}\text { United } \\
\text { States (by } \\
\text { incidence) }\end{array}$ & [6] & $2000-2012$ & 64971 & $\begin{array}{l}\text { Lung } \\
(1.49 \%)\end{array}$ & $\begin{array}{l}\text { Small Intestine } \\
(1.05 \%)\end{array}$ & Rectum (1.04\%) \\
\hline
\end{tabular}

${ }^{1}$ Values in parentheses are $\%$ of study cohort. 
Table 4: Five-year overall survival (OS) rate of NET by primary site (\%)

\begin{tabular}{|c|c|c|c|c|c|c|c|c|}
\hline Country & Authors & Rectum & Lung & Stomach & Pancreas & Colon & $\begin{array}{c}\text { Small } \\
\text { Intestine }\end{array}$ & Appendix \\
\hline Canada & [25] & 100 & & 70 & & 73 & 70 & 84 \\
\hline Germany & [19] & 50 & & 53 & 52 & 48 & 68 & 86 \\
\hline Norway & [24] & 74 & 54 & 45 & 43 & 41 & 59 & 74 \\
\hline Spain & [39] & 64.1 & & 61.4 & 78.1 & 65.1 & $\begin{array}{l}\text { Duodenum } \\
\text { (89.3), } \\
\text { Jejunum- } \\
\text { Ileum } \\
(83.0)\end{array}$ & 100 \\
\hline Taiwan & [21] & 80.9 & 33.9 & 46.4 & 30.2 & 48.1 & 47.9 & 75.7 \\
\hline $\begin{array}{l}\text { U.S. } \\
\text { (Distant } \\
\text { stage G1/ } \\
\text { G2 only; } \\
\text { diagnosed } \\
\text { from 2000- } \\
\text { 2012) }\end{array}$ & [6] & 28 & 32 & 32 & 50 & 29 & 69 & \\
\hline $\begin{array}{l}\text { U.S. } \\
\text { (reported } \\
\text { by median } \\
\text { OS time in } \\
\text { years) }\end{array}$ & [6] & 24.6 & 5.5 & & 3.6 & & & $>30$ \\
\hline
\end{tabular}

Table 5: Five-year OS rate of NET by grade (\%)

\begin{tabular}{|c|c|c|c|c|}
\hline Country & Authors & G1 & G2 & G3 \\
\hline $\begin{array}{l}\text { Argentina } \\
\text { (GEP-NET only; } \\
\text { values were estimated } \\
\text { from graph) }\end{array}$ & [9] & $>75$ & $50-75$ & $<25$ \\
\hline $\begin{array}{l}\text { China } \\
\text { (NET with liver } \\
\text { metastasis only) }\end{array}$ & [32] & $\begin{array}{c}55.6 \\
\text { (surgical resected } \\
\text { patients); } \\
14.3 \text { (non-surgically } \\
\text { resected patients) }\end{array}$ & $\begin{array}{c}35.3 \\
\text { (surgical resected } \\
\text { patients); } \\
0 \text { (non-surgically } \\
\text { resected patients) }\end{array}$ & $\begin{array}{c}28.6 \\
\text { (surgical resected } \\
\text { patients); } \\
0 \text { (non-surgically } \\
\text { resected patients) }\end{array}$ \\
\hline $\begin{array}{l}\text { Korea } \\
\text { (GEP-NET only) }\end{array}$ & [14] & 94.2 & 70.38 & 42.96 \\
\hline $\begin{array}{l}\text { Netherlands (based on } \\
\text { the data from 2001- } \\
\text { 2010) }\end{array}$ & [21] & 80 & 63 & $\begin{array}{c}20 \text { (G3-LCNEC), } \\
6 \text { (G3-SCNEC) }\end{array}$ \\
\hline $\begin{array}{l}\text { Spain } \\
\text { (GEP-NET only) }\end{array}$ & [39] & 83.3 & 77.1 & 43.5 \\
\hline $\begin{array}{l}\text { U.S. } \\
\text { (reported by median } \\
\text { OS time) }\end{array}$ & [6] & 16.2 years & 8.3 years & $\begin{array}{l}10 \text { months (including } \\
\text { G4) }\end{array}$ \\
\hline
\end{tabular}


Table 6: Percentages of NET subtypes categorized according to WHO2010 criteria, reported by country

\begin{tabular}{|c|c|c|c|c|c|}
\hline Country & Author & G1 & G2 & G3 & $\begin{array}{l}\text { Others (if } \\
\text { applicable) }\end{array}$ \\
\hline $\begin{array}{l}\text { Argentina (GEP- } \\
\text { NET only) }\end{array}$ & [9] & $29.5 \%$ & $35.9 \%$ & $9.0 \%$ & No data $(25.6 \%)$ \\
\hline Brazil & {$[31]$} & $73.2 \%$ & $10.5 \%$ & $16.3 \%$ & \\
\hline China & [13] & $3.1 \%$ & $18.0 \%$ & $73.9 \%$ & MANEC (9.1\%) \\
\hline China & {$[32]$} & $27.3 \%$ & $51.5 \%$ & $21.2 \%$ & \\
\hline China & [33] & $29.5 \%$ & $27.3 \%$ & $43.2 \%$ & \\
\hline $\begin{array}{l}\text { China } \\
\text { (Hong Kong) }\end{array}$ & [34] & $87.3 \%$ & NA & NA & \\
\hline Germany & {$[35]$} & $40.4 \%$ & $51.1 \%$ & $18.4 \%$ & \\
\hline Korea & {$[14]$} & $92.31 \%$ & $4.85 \%$ & $2.84 \%$ & \\
\hline Lebanon & {$[37]$} & $56.2 \%$ & $11.2 \%$ & $20.2 \%$ & MANEC (12.4\%) \\
\hline Mexico & {$[38]$} & $64 \%$ & $13 \%$ & $23 \%$ & \\
\hline Netherlands & [21] & $17 \%$ & $1 \%$ & $\begin{array}{l}\text { G3-LCNEC (7\%) } \\
\text { G3-SCNEC (75\%) }\end{array}$ & \\
\hline Norway & [11] & $53.4 \%$ & $24.0 \%$ & $19.6 \%$ & \\
\hline Spain & [39] & $44.4 \%$ & $37.8 \%$ & $17.8 \%$ & \\
\hline U.S. & [6] & $51.0 \%$ & $16.4 \%$ & $\begin{array}{l}32.5 \% \text { (including } \\
\text { G4) }\end{array}$ & \\
\hline
\end{tabular}

NET incidences of KCR and SEER followed a possitive trend over time

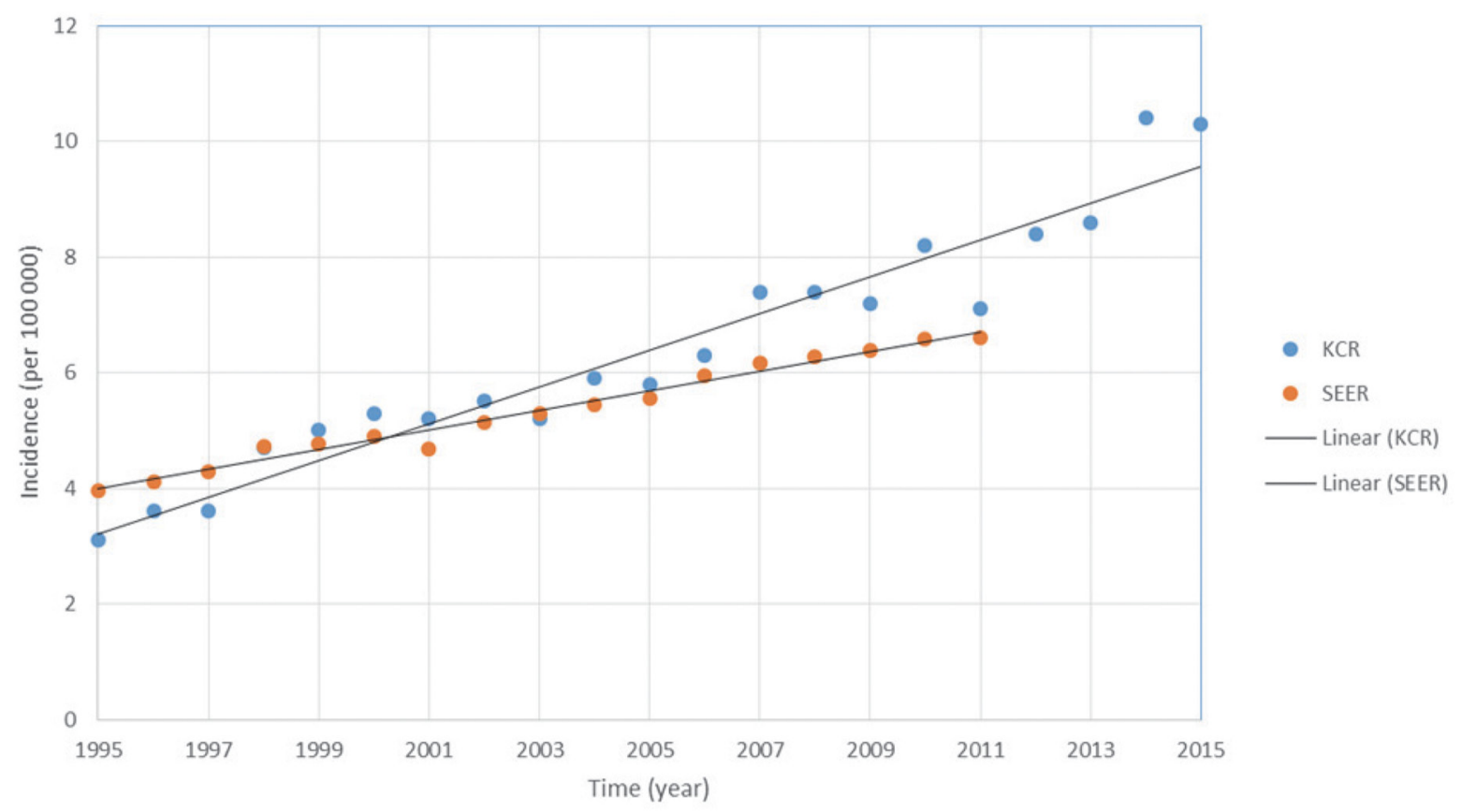

Figure 1: NET incidence according to KCK (blue/series 1) and SEER (red/series 2) [6]. 
different epidemiology studies overtime. Most countries do not have a nation-wide, population based NET registries. Single-center or regional studies cannot assess national incidence and prevalence convincingly.

\section{MATERIALS AND METHODS}

$\mathrm{KCR}$ is a part of NCI's SEER program and has been collecting population-based cancer data since 1986 . We sought Institutional Review Board approval prior to commencement of our study, then proceeded to review the KCR and SEER databases for all newly diagnosed neuroendocrine tumors in Kentucky between 1995 and 2015. Incidence data were adjusted for population. Multivariate analysis was performed using SPSS to analyze various demographic and disease-specific study variables. Tumors were characterized by primary sites. Grading was based on the globally-accepted WHO criteria, which considers $\mathrm{Ki} 67<2 \%$ low grade (G1), 2-20\% intermediate grade $(\mathrm{G} 2),>20 \%$ high grade $(\mathrm{G} 3)$ [1].

Studies outside of the United States (U.S.) were reviewed according to search results from PubMed and Google Scholar. Examples of key words included: "neuroendocrine+tumor"; "neuroendocrine+neoplasm"; "neuroendocrine+prevalence"; "neuroendocrine+incidence";" neuroendocrine+burden"; "neuroendocrine+epidemiology"; "carcinoid+tumor"; "carcinoid+incidence. Studies were sorted into tables according to authors, date, sample size, primary sites of tumor, grading, survival. Re-calculations were performed in some cases based on data from original studies in order to exclude unknown type and to obtain percentages of each tumor type and grade.

\section{CONCLUSIONS}

NETs incidence between 1995 and 2015 showed a linear increase in both KCR and SEER databases. NET incidence has undoubtedly increased over the past two decades. It is imperative for community oncologists to familiarize themselves with this entity, which until recently was under-studied and without many viable treatment options.

\section{ACKNOWLEDGMENTS}

This research was supported by the Cancer Research Informatics Shared Resource of the University of Kentucky Markey Cancer Center (P30CA177558). Authors would like to acknowledge Donna Gilbreath and Cathy Anthony for assistance in preparation of this manuscript.

\section{CONFLICTS OF INTEREST}

The authors declare no conflicts of interest.

\section{REFERENCES}

1. Bosman FT. WHO classification of tumours of the digestive system. World Health Organization, International Agency for Research on Cancer. 2010.

2. Tang LH, Untch BR, Reidy DL, O'Reilly E, Dhall D, Jih L, Basturk O, Allen PJ, Klimstra DS. Well-differentiated neuroendocrine tumors with a morphologically apparent high-grade component: A pathway distinct from poorly differentiated neuroendocrine carcinomas. Clin Cancer Res. 2016; 22: 1011-1017.

3. Yao JC, Hassan M, Phan A, Dagohoy C, Leary C, Mares JE, Abdalla EK, Fleming JB, Vauthey JN, Rashid A, Evans DB. One hundred years after "carcinoid": Epidemiology of and prognostic factors for neuroendocrine tumors in 35,825 cases in the United States. J Clin Oncol. 2008; 26: 3063-3072.

4. Modlin IM, Champaneria MC, Chan AK, Kidd M. A threedecade analysis of 3,911 small intestinal neuroendocrine tumors: The rapid pace of no progress. Am J Gastroenterol. 2007; 102: 1464-1473.

5. Fraenkel M, Kim MK, Faggiano A, Valk GD. Epidemiology of gastroenteropancreatic neuroendocrine tumours. Best Pract Res Clin Gastroenterol. 2012; 26: 691-703.

6. Dasari A, Shen C, Halperin D, Zhao B, Zhou S, Xu Y, Shih $\mathrm{T}$, Yao JC. Trends in the incidence, prevalence, and survival outcomes in patients with neuroendocrine tumors in the United States. JAMA Oncol. 2017; 3:1335-42.

7. Westergaard T, Frisch M, Melbye M. Carcinoid tumors in Denmark 1978-1989 and the risk of subsequent cancers. A population-based study. Cancer. 1995; 76: 106-109.

8. Riihimäki M, Hemminki A, Sundquist K, Sundquist J, Hemminki K. The epidemiology of metastases in neuroendocrine tumors. Int J Cancer. 2016; 139: 2679-2686.

9. O'Connor JM, Marmissolle F, Bestani C, Pesce V, Belli S, Dominichini E, Mendez G, Price P, Giacomi N, Pairola A, Loria FS, Huertas E, Martin C, et al. Observational study of patients with gastroenteropancreatic and bronchial neuroendocrine tumors in Argentina: Results from the large database of a multidisciplinary group clinical multicenter study. Mol Clin Oncol. 2014; 2: 673-684.

10. Lombard-Bohas C, Mitry E, O'Toole D, Louvet C, Pillon D, Cadiot G, Borson-Chazot F, Aparicio T, Ducreux M, Lecomte T. Thirteen-month registration of patients with gastroenteropancreatic endocrine tumours in France. Neuroendocrinology. 2009; 89: 217-222.

11. Sandvik OM, Søreide K, Gudlaugsson E, Kvaløy JT, Søreide JA. Epidemiology and classification of gastroenteropancreatic neuroendocrine neoplasms using current coding criteria. Br J Surg. 2016; 103: 226-232.

12. Ito T, Igarashi $H$, Nakamura $K$, Sasano H, Okusaka T, Takano K, Komoto I, Tanaka M, Imamura M, Jensen RT. Epidemiological trends of pancreatic and gastrointestinal neuroendocrine tumors in Japan: a nationwide survey analysis. J Gastroenterol. 2015; 50: 58-64. 
13. Guo LJ, Wang CH, Tang CW. Epidemiological features of gastroenteropancreatic neuroendocrine tumors in Chengdu city with a population of 14 million based on data from a single institution. Asian Pac J Clin Oncol. 2016; 12: 284-288.

14. Cho MY, Kim JM, Sohn JH, Kim MJ, Kim KM, Kim WH, Kim H, Kook MC, Park DY, Lee JH. Current trends of the incidence and pathological diagnosis of gastroenteropancreatic neuroendocrine tumors (GEP-NETs) in Korea 2000-2009: multicenter study. Cancer Res Treat. 2012; $44: 157$.

15. Tsai HJ, Wu CC, Tsai CR, Lin SF, Chen LT, Chang JS. The epidemiology of neuroendocrine tumors in Taiwan: A nation-wide cancer registry-based study. PLoS One. 2013; 8: e62487.

16. Stefanov R, Miteva-Katrandjieva T, Iskrov G, Damyanov D, Korukov B, Kermedchiev M, Terziev I, Madjov R, Ivanov K, Kolev N, Chenopolski P, Tsaneva M, Dimitrova $\mathrm{V}$, et al. Epidemiological registry of neuroendocrine tumors in Bulgaria--a pilot survey. Khirurgiia (Sofiia). 2014; 7-13.

17. van der Zwan JM, Trama A, Otter R, Larranaga N, Tavilla A, Marcos-Gragera R, Dei Tos AP, Baudin E, Poston G, Links T, Rarecare WG. Rare neuroendocrine tumours: Results of the surveillance of rare cancers in Europe project. Eur J Cancer. 2013; 49: 2565-2578.

18. Tsikitis VL, Wertheim BC, Guerrero MA. Trends of incidence and survival of gastrointestinal neuroendocrine tumors in the United States: a seer analysis. J Cancer. 2012; 3: 292-302.

19. Scherübl H, Streller B, Stabenow R, Herbst H, Höpfner M, Schwertner C, Steinberg J, Eick J, Ring W, Tiwari K. Clinically detected gastroenteropancreatic neuroendocrine tumors are on the rise: epidemiological changes in Germany. World J Gastroenterol. 2013; 19: 9012.

20. Fanello S, Mangone L, Sacchettini C, Vicentini M, Pinto C, and AIRTUM WORKING GROUP. Variety of hormones produced by rare neuroendocrine tumors (NETs): Examination of epidemiology and prognostic factors for NETs in Italy. Journal of Clinical Oncology. 2016; 34:e15656.

21. Korse CM, Taal BG, van Velthuysen ML, Visser O. Incidence and survival of neuroendocrine tumours in the Netherlands according to histological grade: experience of two decades of cancer registry. Eur J Cancer. 2013; 49: 1975-1983.

22. Niederle MB, Hackl M, Kaserer K, Niederle B. Gastroenteropancreatic neuroendocrine tumours: the current incidence and staging based on the WHO and European Neuroendocrine Tumour Society classification: An analysis based on prospectively collected parameters. Endocr Relat Cancer. 2010; 17: 909-918.

23. Hallet J, Law CH, Cukier M, Saskin R, Liu N, Singh S. Exploring the rising incidence of neuroendocrine tumors: A population-based analysis of epidemiology, metastatic presentation, and outcomes. Cancer. 2015; 121: 589-597.
24. Hauso O, Gustafsson BI, Kidd M, Waldum HL, Drozdov I, Chan AK, Modlin IM. Neuroendocrine tumor epidemiology. Cancer. 2008; 113: 2655-2664.

25. McMullen T, Al-Jahdali A, de Gara C, Ghosh S, McEwan A, Schiller D. A population-based study of outcomes in patients with gastrointestinal neuroendocrine tumours. Can J Surg. 2017; 60: 192.

26. Berge T, Linell F. Carcinoid tumours. APMIS. 1976; 84: 322-330.

27. Godwin JD. Carcinoid tumors an analysis of 2837 cases. Cancer. 1975; 36: 560-569.

28. Hemminki K, Li X. Incidence trends and risk factors of carcinoid tumors. Cancer. 2001; 92: 2204-2210.

29. Janson ET, Holmberg L, Stridsberg M, Eriksson B, Theodorsson E, Wilander E, Öberg K. Carcinoid tumors: analysis of prognostic factors and survival in 301 patients from a referral center. Ann Oncol. 1997; 8: 685-690.

30. Newton J, Swerdlow A, dos Santos Silva I, Vessey M, Grahame-Smith D, Primatesta P, Reynolds D. The epidemiology of carcinoid tumours in England and Scotland. Br J Cancer. 1994; 70: 939.

31. Estrozi B, Bacchi CE. Neuroendocrine tumors involving the gastroenteropancreatic tract: A clinicopathological evaluation of 773 cases. Clinics. 2011; 66: 1671-1675.

32. Du S, Wang Z, Sang X, Lu X, Zheng Y, Xu H, Xu Y, Chi T, Zhao H, Wang W. Surgical resection improves the outcome of the patients with neuroendocrine tumor liver metastases: large data from Asia. Medicine (Baltimore). 2015; 94:e388.

33. Zhang $\mathrm{M}$, Zhao $\mathrm{P}$, Shi $\mathrm{X}$, Zhao A, Zhang L, Zhou L. Clinicopathological features and prognosis of gastroenteropancreatic neuroendocrine neoplasms in a Chinese population: A large, retrospective single-centre study. BMC Endocr Disord. 2017; 17: 39.

34. Chan DT, Luk AO, So W, Kong AP, Chow FC, Ma RC, Lo AW. Natural history and outcome in chinese patients with gastroenteropancreatic neuroendocrine tumours:-a 17-year retrospective analysis. BMC Endocr Disord. 2016; 16: 12.

35. Ploeckinger U, Kloeppel G, Wiedenmann B, Lohmann R. The German NET-Registry: An audit on the diagnosis and therapy of neuroendocrine tumors. Neuroendocrinology. 2009; 90: 349-363.

36. Faggiano A, Ferolla P, Grimaldi F, Campana D, Manzoni M, Davi M, Bianchi A, Valcavi R, Papini E, Giuffrida D. Natural history of gastro-entero-pancreatic and thoracic neuroendocrine tumors. Data from a large prospective and retrospective Italian epidemiological study: the NET management study. J Endocrinol Invest. 2012; 35: 817.

37. Kourie HR, Ghorra C, Rassy M, Kesserouani C, Kattan J. Digestive neuroendocrine tumor distribution and characteristics according to the 2010 WHO Classification: A single institution experience in lebanon. Asian Pac J Cancer Prev. 2016; 17: 2679-2681. 
38. Medrano Guzman R. (2017). Clinical and epidemiological features in 495 gastroenteropancreatic neuroendocrine patients in Mexico. Journal of Clinical Oncology. 2017; 35:e15687.

39. Garcia-Carbonero R, Capdevila J, Crespo-Herrero G, Diaz-Perez J, Martínez Del Prado M, Alonso Orduna V,
Sevilla-Garcia I, Villabona-Artero C, Beguiristain-Gómez A, Llanos-Munoz M. Incidence, patterns of care and prognostic factors for outcome of gastroenteropancreatic neuroendocrine tumors (GEP-NETs): Results from the National Cancer Registry of Spain (RGETNE). Ann Oncol. 2010; 21: 1794-1803. 